\title{
Effects of Crystal Shape on the Energy Levels of Zero-Dimensional PbS Quantum Dots
}

\author{
Z. Hens and D. Vanmaekelbergh \\ Debye Institute, Utrecht University, P.O. Box 80000, 3508 TA Utrecht, The Netherlands
}

E. J. A. J. Stoffels and H. van Kempen

Research Institute for Materials, Nijmegen University, Toernooiveld 1, 6525 ED Nijmegen, The Netherlands

(Received 10 December 2001; published 24 May 2002)

\begin{abstract}
Nanometer-size PbS quantum dots have been made by electrodeposition on a $\mathrm{Au}(111)$ substrate. The deposited nanocrystals have a flattened cubic shape. We probed the single-electron energy-level spectrum of individual quantum dots by scanning tunneling spectroscopy and found that it deviates strongly from that of spherical $\mathrm{PbS}$ quantum dots. The measured energy-level spectrum is successfully explained by considering strong confinement in a flattened cubic box.
\end{abstract}

DOI: $10.1103 /$ PhysRevLett.88.236803

PACS numbers: 73.22.-f, 73.63.-b

Insulating nanocrystals with dimensions below $10 \mathrm{~nm}$ can show atomiclike discrete energy levels due to quantum confinement [1]. Such nanocrystals are therefore often called "zero-dimensional quantum dots" or "artificial atoms." The energy-level spectrum depends on the chemical identity and crystal structure of the nanocrystals and on their size and shape. Many II-VI and III-V semiconductors have been prepared as insulating nanocrystals by colloidal chemistry. Nucleation and growth in solution leads to (nearly) spherical crystals [2] — unless the growth conditions are carefully manipulated [3]. In contrast, deposition on a surface (molecular beam epitaxy, electrodeposition) typically yields nonspherical structures [4-6]. The question as to how far the energy level spectrum of very small (zero-dimensional) quantum dots is affected by the shape of the nanocrystals has not been addressed systematically. However, as is clear from recent optical work on insulating quantum rods [3], relatively small deviations from spherical symmetry can have dramatic effects on the optical properties of semiconductor nanocrystals.

In this Letter, we report on a study of the energy-level spectrum of electrodeposited $\mathrm{PbS}$ "quantum boxes," which show a flattened cubic shape ( $x: y: z$ typically between $2: 2: 1$ and 3:3:1, $x$ and $y$ being parallel and $z$ being perpendicular to the substrate surface). Lead chalcogenide nanocrystals could form the ultimate quantum dots due to the low effective mass of both electrons and holes, ensuring strong confinement $[7,8]$. We probed the energy levels of a number of individual quantum dots, deposited on $\mathrm{Au}(111)$, with electron tunneling spectroscopy at $4.2 \mathrm{~K}$. It is found that the quasiparticle gap is significantly larger, and the energylevel separation is significantly smaller than experimentally observed and theoretically predicted for spherical $\mathrm{PbS}$ dots [7]. The measured energy-level spectrum of the flattened cubic $\mathrm{PbS}$ quantum box is successfully explained by discriminating between the (strong) quantization perpendicular to, and the (weak) quantization parallel to, the gold surface. For the first time, it is experimentally shown that the energy-level spectrum of so-called zero-dimensional quantum dots can be sensitive to deviations from a spherical shape.

We deposited $\mathrm{PbS}$ nanocrystals from an aqueous solution ( $1 \mathrm{mM})$ of $\mathrm{Pb}\left(\mathrm{NO}_{3}\right)_{2}$ and $\mathrm{Na}_{2} \mathrm{~S}_{2} \mathrm{O}_{3}$ at $p \mathrm{H} 2.8$ [9] on flame-annealed $\mathrm{Au}(111)$, graphite (HOPG), and $\mathrm{Au}(111)$ covered with a 1,4-dithiane self-assembled monolayer (SAM) (Au |SAM). The growth of crystalline PbS was confirmed by $\mathrm{x}$-ray diffraction analysis of a thin layer $(1 \mu \mathrm{m})$, grown on $\mathrm{Au}(111)$. Individual nanocrystals could be formed by depositing a layer of a few nanometers in thickness. A TEM study demonstrated the formation of $\mathrm{PbS}$ nanocrystals on $\mathrm{Au}(111)$. The size of the $\mathrm{PbS}$ nanocrystals could be controlled by the charge used for deposition.

We have investigated more than 10 isolated $\mathrm{PbS}$ nanocrystals with room-temperature scanning tunneling microscopy (STM) and atomic force microscopy (AFM), with dimensions in the 10 to $100 \mathrm{~nm}$ range. Figure 1 shows an AFM image of a $\mathrm{PbS}$ nanocrystal grown on

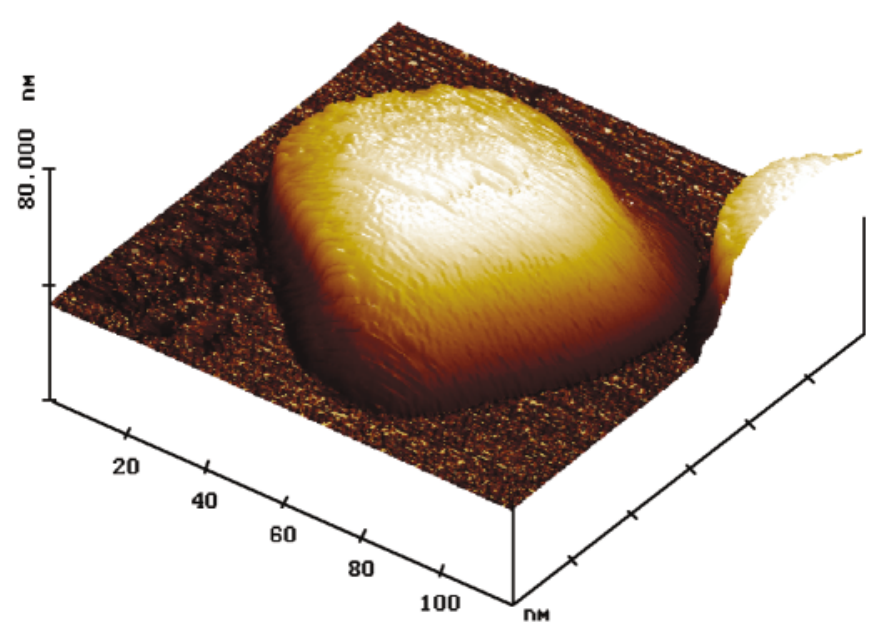

FIG. 1 (color). Tapping mode AFM image $\left(125 \times 125 \mathrm{~nm}^{2}\right)$ of a single PbS nanocrystal deposited on HOPG. The nanocrystal is a flattened cube that measures 60:60:25 $\mathrm{nm}$. 
HOPG. It is sufficiently large to allow for a full characterization by AFM. The nanocrystal is a flattened cube having - after correcting for the finite width of the tip - relative dimensions $(x: y: z)$ of 60:60:25 (nm). This shape complies with the cubic rocksalt structure of crystalline $\mathrm{PbS}$. Similar nanocrystals, 15 to $25 \mathrm{~nm}$ in lateral size, are obtained by PbS deposition on Au |SAM. Most nanocrystals investigated have a flattened cubic shape; the aspect ratio (lateral vs perpendicular size) ranges between 2 and 3.

Figure 2 shows a low-temperature STM image of an individual $\mathrm{PbS}$ nanocrystal on $\mathrm{Au}(111)$. The nanocrystal is well separated from its neighbors. The top surface is squarelike and flat on an atomic scale $(0.1 \mathrm{~nm}$ rms roughness). Therefore, its dimensions $(7.5$ by $7.5 \mathrm{~nm}$ ) give a reliable estimate of the true (lateral) size of the quantum dot. The apparent height obtained from the STM image is about $2 \mathrm{~nm}$. Although STM provides an estimate of the real height only, we can conclude that also the small nanocrystals have a flattened cubic shape (tip convolution reduces the steepness of its sides).

To study the energy-level spectrum of individual $\mathrm{PbS}$ nanocrystals, we used scanning tunneling spectroscopy [10,11]. For this purpose, an STM tip is placed above a quantum dot attached to a conducting substrate and the tunneling current $I$ is measured as a function of the tipsubstrate potential difference (bias) $V$. Since current flow results from resonant tunneling via the quantum dot orbitals, a plot of the differential conductance $(d I / d V)$ as a function of $V$ (tunneling spectrum) shows a sequence of peaks, reflecting the electron and hole energy levels in the quantum dot. In the present study, tunneling spectra are obtained by digitally filtering and differentiating the experimental $I-V$ curves. All STM work was performed in a liquid helium cryostat with an electrochemically etched $\operatorname{Pt}(90 \%): \operatorname{Ir}(10 \%)$ tip. The measurements were repeated on three different $\mathrm{Au}$ substrates on which $\mathrm{PbS}$ nanocrystals of

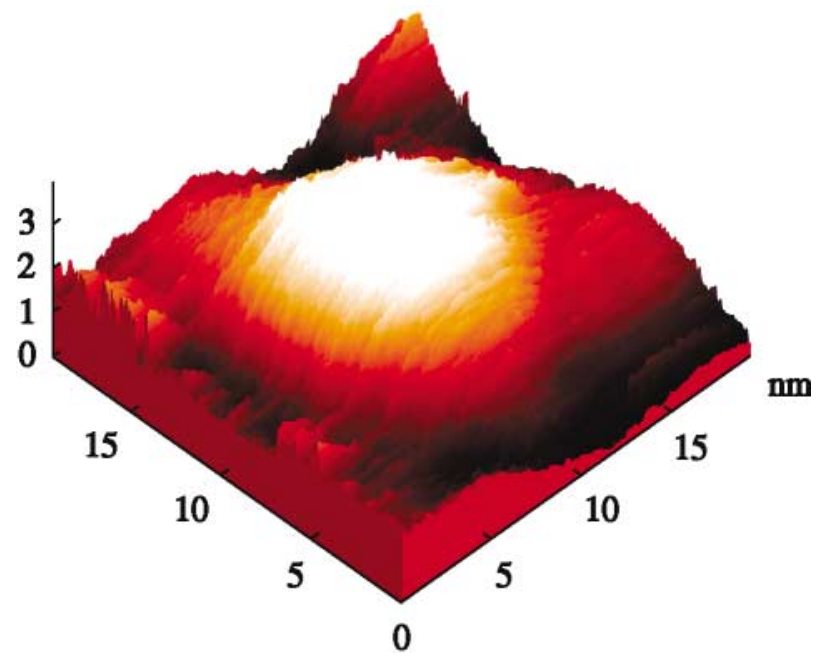

FIG. 2 (color). STM image $\left(19 \times 19 \mathrm{~nm}^{2}\right)$ of a single isolated $\mathrm{PbS}$ nanocrystal deposited on $\mathrm{Au}(111)$. a given size were deposited. On each nanocrystal, at least 25 identical $I-V$ curves were obtained.

In Fig. 3, tunneling spectra of $\mathrm{PbS}$ quantum dots of two different sizes are shown. The top surface (STM topography, $10 \%$ error) measures 10 by $10 \mathrm{~nm}$ for the larger and 4 by $4 \mathrm{~nm}$ for the smaller particles. The nanocrystals will be referred to by their lateral size (10 and $4 \mathrm{~nm})$. The spectra obtained for the $10 \mathrm{~nm}$ particles (Fig. 3A) show a quasiparticle gap $\Delta E^{q p}$ of $0.68 \pm 0.02 \mathrm{eV}$. For these spectra, three conductance peaks could be systematically identified at positive bias, with level separations of $0.16 \pm 0.02 \mathrm{eV}$ $\left(\Delta_{1-2}^{e}\right)$ and $0.27 \pm 0.02 \mathrm{eV}\left(\Delta_{2-3}^{e}\right)$, whereas only a single peak was repeatedly observed at negative bias. In the case of the $4 \mathrm{~nm}$ particles (Fig. 3B), we found a quasiparticle gap of $2.35 \pm 0.1 \mathrm{eV}$. Two major conductance peaks, separated by $0.89 \pm 0.03 \mathrm{eV}\left(\Delta_{1-2}^{e}\right)$ could be identified at positive bias. At negative bias, two small peaks separated by $0.24 \pm 0.03 \mathrm{eV}\left(\Delta^{h}\right)$ were observed. The lower intensity of the latter peaks is indicative of tunneling through valence-band levels [11]. Figure 4 shows a detail of the first and the second conductance peak at positive bias for two spectra, obtained with a $4 \mathrm{~nm}$ PbS nanocrystal. A fine structure with, at the most, four approximately equally spaced peaks can be observed between the two major peaks. The spacing $\Delta^{e}$ between the fine-structure levels is $0.18 \pm 0.02 \mathrm{eV}$. For the $10 \mathrm{~nm}$ nanocrystals, this fine structure is not systematically observed, although in some cases additional peaks were found between the main peaks.

As demonstrated by Fig. 4, the relative intensity of the fine-structure peaks of the $4 \mathrm{~nm}$ quantum dots varies considerably from spectrum to spectrum. We checked whether

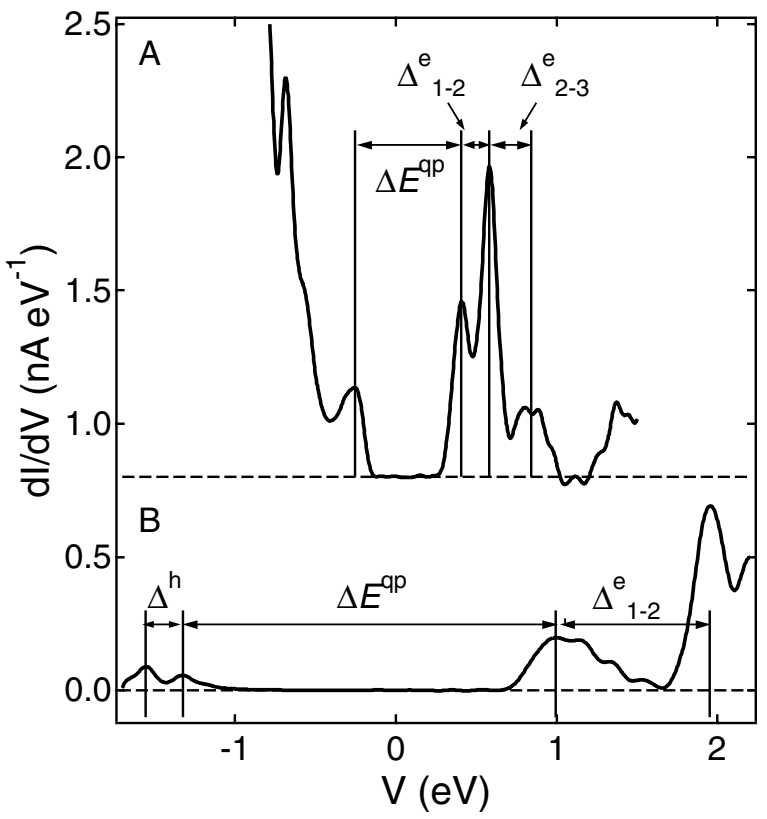

FIG. 3. Tunneling spectra obtained at $4.2 \mathrm{~K}$ at electrodeposited $\mathrm{PbS}$ quantum dots of two different sizes [(A) $10 \mathrm{~nm}$; (B) $4 \mathrm{~nm}$ ]. Symbols are explained in the text. 


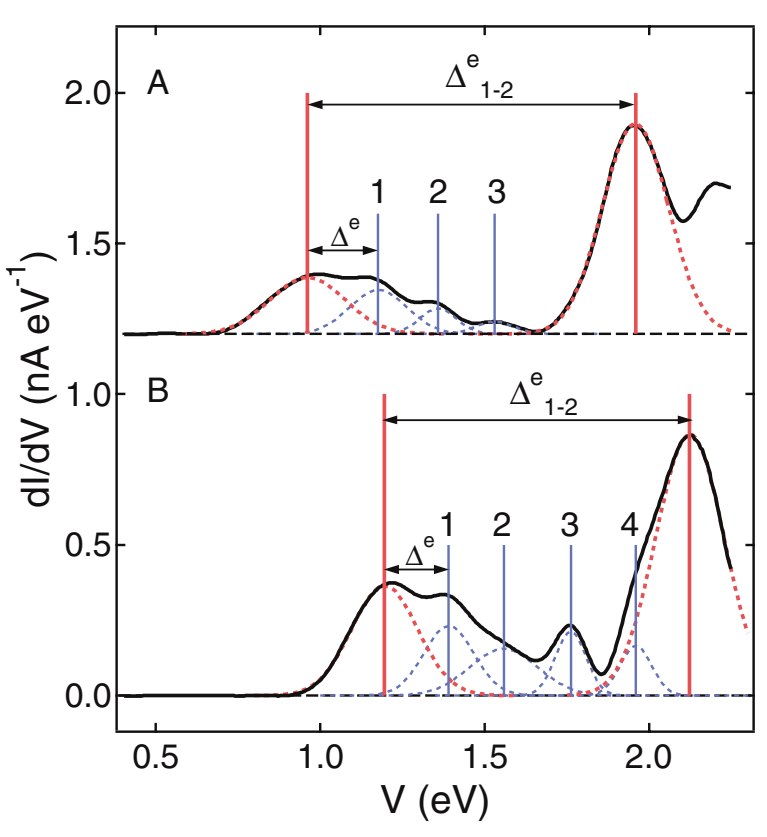

FIG. 4 (color). Details of the conductance peaks at positive bias measured on a $4 \mathrm{~nm} \mathrm{PbS}$ quantum dot. Two experiments on the same nanocrystal are presented (the zero line is shifted upwards in the case of spectrum A). Apart from the two main peaks (red), up to four fine-structure peaks (blue) follow the first main peak. The separation $\Delta^{e}$ between these peaks is $0.18 \pm 0.02 \mathrm{eV}$. It is shown that the intensity of the different fine-structure peaks may vary considerably.

this fine structure is related to electron-electron Coulomb repulsion by varying the rate of tunneling into, with respect to tunneling out of, the dot. No systematic relation between the relative intensity of the conductance peaks and the rate of tunneling into the quantum dot was found. We infer that effects of electron-electron interactions (e.g., Coulomb blockade) on the tunneling spectrum can be neglected [11]. The tunneling spectra are shell-tunneling spectra (i.e., one electron at the time tunnels through the quantum dot), which reflect the single-particle energy levels of the quantum dot. This stems with the fact that the $\mathrm{Au}(111) \mid \mathrm{PbS}$ barrier is very thin, thus ensuring that tip-to-dot tunneling is much slower than dot-to-gold tunneling. Furthermore, the optical gap of the $4 \mathrm{~nm}$ quantum dots (derived from the reflectance spectrum) is $2.3 \pm 0.2 \mathrm{eV}$, as large as the quasiparticle gap in the tunneling spectrum. Hence, the electron and hole charging energies are strongly screened by the gold electrons [12].

In Table I, the experimental data obtained for the flattened cubic $4 \mathrm{~nm}$ quantum dots are compared with theoretical results of spherical $\mathrm{PbS}$ quantum dots [7]. The latter data were shown to agree well with optical absorption measurements on spherical $\mathrm{PbS}$ quantum dots [7]. Since the tip | dot | gold barrier is highly asymmetric in the case of electrodeposited quantum dots, the experimental data are taken directly from the measured tunneling spectra [13]. For equal volume quantum dots, Table I shows a striking inconsistency: Whereas the quasiparticle gap is lower by
TABLE I. Comparison of the quasiparticle gap $\Delta E^{q p}$ and the level separations $\Delta^{e}$ and $\Delta^{h}$ as obtained experimentally at the $4 \mathrm{~nm}$ quantum dots and the theoretical values for spherical quantum dots of equal volume [7].

\begin{tabular}{lccc}
\hline \hline Dot type & $\Delta E^{q p} / \mathrm{eV}$ & $\Delta^{e} / \mathrm{eV}$ & $\Delta^{h} / \mathrm{eV}$ \\
\hline Experiment & $2.35 \pm 0.1$ & $0.18 \pm 0.02$ & $0.24 \pm 0.03$ \\
Theory & $1.55 \pm 0.05$ & $0.45 \pm 0.05$ & $0.55 \pm 0.05$ \\
\hline \hline
\end{tabular}

a factor of about 1.5 for a spherical quantum dot, the level separations $\Delta^{e}$ and $\Delta^{h}$ are higher by a factor of about 2.5 . In other words, a spherical PbS quantum dot cannot combine a quasiparticle gap of $2.35 \mathrm{eV}$ with level separations of 0.18 and $0.24 \mathrm{eV}$ for electrons and holes, respectively. Thus, the energy-level spectrum obtained with flattened cubic $\mathrm{PbS}$ quantum dots differs profoundly from that of spherical $\mathrm{PbS}$ quantum dots.

Figure 5 shows the variation of the lowest-lying energy levels of a flattened cube of constant volume, as a function of the aspect ratio, calculated by a quasiparticle in a box approximation (infinite potential barriers). Since the energy levels of a sphere and a true cube are in close agreement [14], the case of a spherical quantum dot corresponds to an aspect ratio of one. An increase of the aspect ratio reduces the symmetry of the nanocrystal; this induces

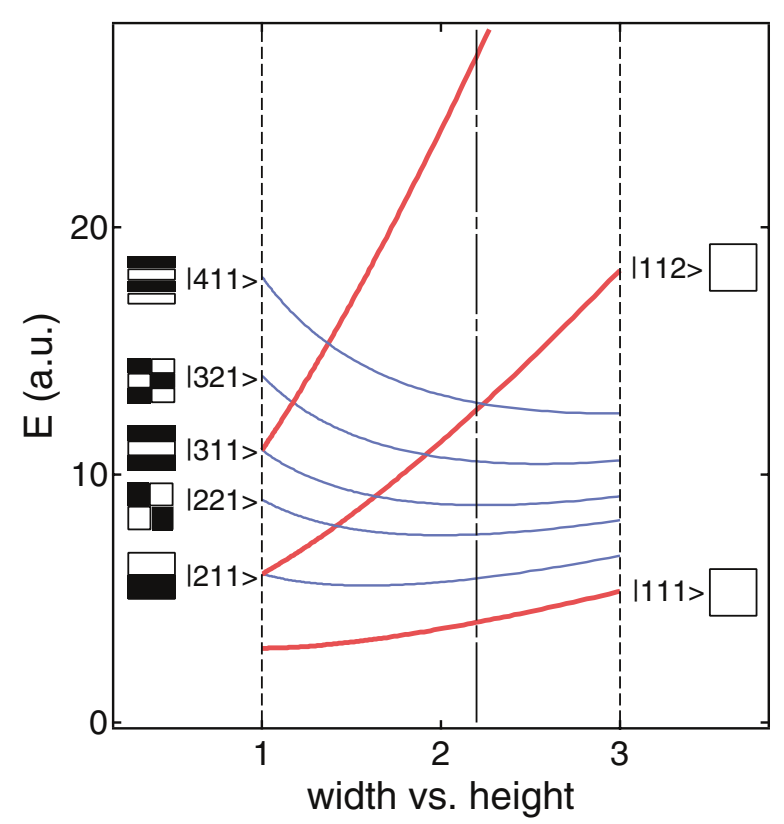

FIG. 5 (color). Variation of the energy levels in a flattened cubic quantum box as a function of the width to height ratio. The eigenenergies are characterized by the quantum numbers $n_{x}$ and $n_{y}$ (parallel quantization) and $n_{z}$ (perpendicular quantization). Eigenenergies of states having $n_{x}=n_{y}=1$ are drawn in red, those with $n_{z}=1$ in blue. For every eigenstate depicted, the projection of the orbital on the $x-y$ plane (parallel to the substrate surface) is depicted, indicating regions where the sign of the wave function is negative or positive in black or white, respectively (degenerate states are shown only once). The dash-dotted line corresponds to an aspect ratio of 2.2. 
a distinction between the quantization parallel ( $x$ and $y$ direction) and perpendicular ( $z$ direction) to the substrate surface. Figure 5 demonstrates that the energy difference between states having a constant quantum number $n_{z}$ (parallel quantization, blue line) decreases with increasing aspect ratio, whereas that between states having constant quantum numbers $n_{x}$ and $n_{y}$ (perpendicular quantization, red line) increases. Moreover, as depicted schematically in Fig. 5, all the orbitals show nodal planes perpendicular to the gold surface, except states with $n_{x}=1$ and $n_{y}=1$. As the overlap between electron states in the tip and in the quantum dot determines the tunneling rate, tunneling to a given state will be suppressed if the tip is above a node. Hence, only tunneling to states with $n_{x}=1$ and $n_{y}=1$ (red lines) will be largely insensitive to the position of the tip relative to the quantum dot. The intensity of the other peaks may vary considerably, depending on the position of the tip relative to the nodes of the orbitals.

The experiments on the $4 \mathrm{~nm}$ quantum dots show four fine-structure peaks of varying intensity between two major peaks of constant intensity. Figure 5 shows that four, almost equally spaced energy levels with quantum number $n_{z}=1$ are present between the LUMO $\left(\mid n_{x}=1, n_{y}=\right.$ $\left.\left.1, n_{z}=1\right\rangle\right)$ and the first level with $n_{z}=2(|112\rangle)$, when the aspect ratio is 2.2. Therefore, we conclude first of all that the two main peaks (red in Fig. 4) in the tunneling spectra of the $1.6 \mathrm{~nm}$ quantum dots are due to tunneling to the $|111\rangle$ and the $|112\rangle$ states (perpendicular quantization, red lines in Fig. 5). As indicated above, the level separation between these states increases strongly with increasing aspect ratio. The peak assignment complies with this trend: The energy difference of $0.89 \mathrm{eV}$ found experimentally between the $|111\rangle$ and the $|112\rangle$ states is twice the separation of $0.45 \mathrm{eV}$ between the first two levels of an equal-volume spherical dot (i.e., the energy separation expected between these states for an aspect ratio of 1). Next, we infer that the fine-structure peaks (blue in Fig. 4) originate from tunneling to states having $n_{z}=1$ and $n_{x}$ and $n_{y}$ equal to either 2 or 3 (parallel quantization, blue lines in Fig. 5). Hence, the unexpectedly low value of the level separation found experimentally can be attributed to the relatively weak quantization parallel to the gold surface. A similar conclusion holds at negative bias: The two peaks found in the tunneling spectra of the $1.6 \mathrm{~nm}$ quantum dots are related to tunneling to the $|111\rangle$ and the $|211\rangle$ hole states, respectively. The higher level separation $(0.24 \mathrm{eV}$ vs $0.18 \mathrm{eV})$ agrees with the fact that the effective mass of holes is slightly smaller than that of electrons in $\mathrm{PbS}$ [15]. In the case of the $10 \mathrm{~nm} \mathrm{PbS}$ nanocrystals, fine structure could not be observed, given the width of the conductance peaks. The major conductance peaks may then be identified with tunneling to levels with increasing quantum number $n_{z}$ (red lines in Fig. 5). Using the energy values depicted in Fig. 5, we find a ratio $\Delta_{1-2}^{e} / \Delta_{2-3}^{e}$ of $1: 1.6$, in close agreement with the experimentally observed value of $16: 27$.

In summary, we have demonstrated strong size quantization in individual electrodeposited $\mathrm{PbS}$ nanocrystals. As these structures are flattened cubes, two quantization lengths determine the single-electron energy-level spectrum. The overall structure of the single-electron energy-level spectrum is governed by the strong perpendicular quantization. The weaker parallel quantization leads to a fine structure, i.e., a set of closely space eigenstates with constant perpendicular quantum number. We conclude that a relatively small deviation from spherical crystal shape has a pronounced effect on the energy-level spectra of zero-dimensional quantum dots.

Z.H. acknowledges the Fonds voor Wetenschappelijk Onderzoek-Vlaanderen (FWO-Vlaanderen) for its financial support. E. J. A.J.S. and H.v. K. acknowledge the Stichting voor Fundamenteel Onderzoek der Materie (FOM) for its financial support.

[1] L. E. Brus, J. Chem. Phys. 80, 4403 (1984).

[2] C. B. Murray, D. J. Norris, and M. G. Bawendi, J. Am. Chem. Soc. 115, 8706 (1993).

[3] J. Hu, L. Liang-shi, W. Yang, L. Manna, W. Lin-wang, and A. P. Alivisatos, Science 292, 2060 (2001).

[4] S. T. Yau and P. G. Velikov, Nature (London) 406, 494 (2000).

[5] B. Grandidier, Y.M. Niquet, B. Legrand, J. P. Nys, C. Priester, D. Stiévenard, J. M. Gérard, and V. ThierryMieg, Phys. Rev. Lett. 85, 1068 (2000).

[6] M. Kleinert, H. F. Waibel, G. E. Engelmann, H. Martin, and D. M. Kolb, Electrochim. Acta 46, 3129 (2001).

[7] I. Kang and F. Wise, J. Opt. Soc. Am. 14, 1632 (1997).

[8] F. Wise, Acc. Chem. Res. 33, 773 (2000).

[9] M. Sharon, K. S. Ramaiah, M. Kumar, M. NeumannSpallart, and C. Lévi-Clément, J. Electroanal. Chem. 436, 49 (1997).

[10] U. Banin, Y. W. Cao, D. Katz, and O. Millo, Nature (London) 400, 542 (1999).

[11] E. P. A. M. Bakkers, Z. Hens, A. Zunger, A. Franceschetti, L. P. Kouwenhoven, L. Gurevich, and D. Vanmaekelbergh, Nano Lett. 1, 551 (2001).

[12] A. Franceschetti, A. Williamson, and A. Zunger, J. Phys. Chem. B 104, 3398 (2000).

[13] E. P. A. M. Bakkers and D. Vanmaekelbergh, Phys. Rev. B 62, R7743 (2000).

[14] P. G. Bolcatto and C. R. Proetto, Phys. Rev. B 59, 12487 (1999).

[15] R. C. Weast, CRC Handbook of Chemistry and Physics (CRC Press, Boca Raton, Florida, 1988), p. E82. 